\title{
Allatostatin-C antagonizes the synergistic myostimulatory effect of allatotropin and serotonin in Rhodnius prolixus (Stal)
}

\author{
María José Villalobos-Sambucaro ${ }^{a}$, Luis Anibal Diambra ${ }^{\mathrm{b}}$, Fernando Gabriel Noriega ${ }^{\mathrm{c}, \mathrm{d}}$, \\ Jorge Rafael Ronderos ${ }^{\mathrm{a}, *}$ \\ ${ }^{a}$ Cátedra Histología y Embriología Animal (FCNyM-UNLP), La Plata, Argentina \\ ${ }^{\mathrm{b}}$ Centro Regional de Estudios Genómicos (CREG-UNLP), La Plata, Argentina \\ ${ }^{\mathrm{c}}$ Department of Biological Sciences, Florida International University, Miami, FL, USA \\ ${ }^{\mathrm{d}}$ Biomolecular Science Institute, Florida International University, Miami, USA
}

\section{A R T I C L E I N F O}

\section{Article history:}

Received 28 December 2015

Revised 4 May 2016

Accepted 8 May 2016

Available online 9 May 2016

\section{Keywords:}

Allatotropin

Allatostatin-C

Rhodnius prolixus

Cardioregulatory

Myoregulatory

Peptides

Neuropeptides

\begin{abstract}
A B S T R A C T
Haematophagous insects can ingest large quantities of blood in a single meal producing a large quantity of urine in the following hours to eliminate the excess of water and mineral ions incorporated. The excretory activity of the Malpighian tubules is facilitated by an increase in haemolymph circulation as a result of the intensification of aorta contractions, combined with an increase of anterior midgut peristaltic waves. We have recently shown that haemolymph circulation during post-prandial diuresis is modulated by the synergistic activity of allatotropin (AT) and serotonin, resulting in an increase in aorta and crop contraction rates. In the present study we describe the antagonistic effect of allatostatin-C (AST-C) on the increase of aorta frequency of contractions induced by serotonin/AT in Rhodnius prolixus. The administration of AST-C counteracted the increase in the frequency induced by the treatment with serotonin/ AT, but did not affect the increase in frequency induced by the administration of serotonin alone, suggesting that AST-C is altering the synergism between serotonin and AT. Furthermore, the administration of AST-C during post-prandial diuresis decreases the number of peristaltic waves of the anterior midgut. The AST-C putative receptor is expressed in the hindgut, midgut and dorsal vessel, three critical organs involved in post-prandial diuresis. All together these findings provide evidence that AST-C plays a key role as a myoregulatory and cardioregulatory peptide in R. prolixus.
\end{abstract}

(c) 2016 Elsevier Inc. All rights reserved.

\section{Introduction}

Juvenile individuals of the kissing bug Rhodnius prolixus (Stal) (Hemiptera: Reduviidae) can ingest a volume of blood up to 12.5 times its unfed weight in a single meal (Buxton, 1930). As a consequence, large volumes of urine are produced during the next few hours to decrease weight, eliminate the excess of water and mineral ions, and restore homeostasis (Ramsay, 1952; Maddrell, 1964, 1978; Maddrell et al., 1993; O'Donnell et al., 2003). To accomplish this process, Malpighian tubules (MTs) respond by increasing their rate of secretion to produce hyposmotic urine that re-establish the osmotic balance (Maddrell, 1964; Maddrell and Phillips, 1975). This physiological process is controlled by diuretic

\footnotetext{
* Corresponding author at: Cátedra Histología Embriología Animal (FCNyMUNLP), Universidad Nacional de La Plata, Calle 64 N³ (1900), La Plata, Buenos Aires, Argentina.

E-mail addresses: jrondero@museo.fcnym.unlp.edu.ar, ronderos@isis.unlp.edu.ar (J.R. Ronderos)
}

and anti-diuretic hormones; serotonin being one of the most important regulator of MTs activity (Maddrell and Phillips, 1975; Maddrell et al., 1991). Water and ion homeostasis also depends on the ability of the dorsal vessel (DV) to pump haemolymph in a posterior-anterior direction (Chiang et al., 1990). Furthermore, $R$. prolixus diuresis also depends on the ability of the anterior midgut (crop) to move haemolymph in an antero-posterior direction (Maddrell, 1964). In fact, almost immediately after the beginning of ingestion of blood, the frequencies of peristaltic waves of the crop and dorsal vessel contractions increase, facilitating the haemolymph recirculation (Maddrell, 1964).

A serotonin receptor was recently isolated and characterized in $R$. prolixus (Paluzzi et al., 2015); where serotonin acts as a diuretic factor and also controls other processes during feeding, including salivation and plasticization of the cuticle (Orchard, 2006), as well as visceral and cardiac muscle contractions (Paluzzi et al., 2015; Villalobos-Sambucaro et al., 2015). Serotonin is also involved in the regulation of visceral and cardiac muscle contractions in Drosophila melanogaster (Dasari and Cooper, 2006), and accelerates the 
heart frequency of contractions in the mosquito Anopheles gambiae (Hillyer et al., 2015). Allatotropin (AT), a neuropeptide isolated on the basis of its activity stimulating the synthesis of juvenile hormones in the lepidopteran Manduca sexta (Kataoka et al., 1989), has proved to be pleiotropic, acting in different insect species as myoregulator and cardioaccelerator (Duve et al., 1999, 2000; Koladich et al., 2002; Rudwall et al., 2000). In Triatoma infestans (Hemiptera: Reduviidae) (the most important vector of Chagas disease in several South American countries), AT increases the frequency of contractions of the DV, crop and hindgut (HG) (Santini and Ronderos, 2007; Sterkel et al., 2010). Moreover, it was shown in unfed adults of $T$. infestans that AT has no myoregulatory effect by itself, but synergizes the stimulatory activity of serotonin on the frequency of the DV contractions (Sterkel et al., 2010). Similarly, in $R$. prolixus, it was shown that AT has no effect on the heart beat frequency, or the contractions of the digestive tract under basal conditions (Masood and Orchard, 2014). However, a recently published study described that, as in T. infestans, a synergistic activity of serotonin and AT is also manifest in $R$. prolixus (Villalobos-Sambucaro et al., 2015). In the same study it was indicated that the putative AT receptor was expressed in the midgut (MG), rectum and DV (organs modulated by AT in triatominae) (Santini and Ronderos, 2007; Sterkel et al., 2010).

Allatostatins (ASTs) are a group of three structurally unrelated families of peptides originally associated with the control of corpora allata (CA) activity (for a review see Bendena and Tobe, 2012). Like AT, ASTs are pleiotropic peptides, having myoregulatory functions in several insect species (Duve et al., 1999, 2000; Matthews et al., 2007; Robertson et al., 2012).

In the present study, we report the expression of a putative AST$C$ receptor in several organs of $R$. prolixus, including heart and DV; and demonstrate that treatment of unfed adult males with AST-C during the period of highest serotonin/AT stimulatory activity results in a decrease of the beat frequency of the aorta. Furthermore, the administration of AST-C during post-prandial diuresis showed a dose-response decrease of the aorta number of contractions, and also of the number of peristaltic waves of the anterior midgut (crop). All together these results suggest that AST-C is involved in the regulation of haemolymph recirculation during diuresis in $R$. prolixus.

\section{Material and methods}

\subsection{Insects}

$R$. prolixus were obtained from a colony maintained at $28 \pm 2{ }^{\circ} \mathrm{C}$, $45 \%$ relative humidity and a $12: 12 \mathrm{~h}$ light-dark period. Experiments were performed with non-fed and recently fed adult male insects. The individuals were immediately isolated after molting (i.e. fifth instar to adult) and starved during 14-21 days. For experiments performed with fed insects, a blood-meal was offered after the starvation period. Only those insects fed ad libitum were used. Experimental groups included 6-12 insects. For mRNA expression analysis, cDNA obtained from female organs were also included.

\subsection{Myoregulatory assays}

The effect of AST-C on the contractions of the aorta and anterior midgut was analyzed in vivo. To perform these experiments, the wings of the insects were removed to expose the dorsal cuticle of the abdomen. Due to the transparent nature of the cuticle, the contractions of the aorta and the peristaltic waves of the anterior midgut were clearly seen and could be recorded (Sterkel et al., 2010; Villalobos-Sambucaro et al., 2015). We tested the effect of Aedes aegypti AT $\left(10^{-9} \mathrm{M}\right)$ and AST-C $\left(10^{-14}, 10^{-12}, 10^{-10}, 10^{-8}\right.$ and
$10^{-6} \mathrm{M}$ ) (Biopeptide, San Diego, CA) (Hernández-Martínez et al., 2005). The sequences of both peptides tested are AT: APFRNSEMMTARGF and AST-C: QIRYRQCYFNPISCF. Peptides were diluted in $3 \mu \mathrm{l}$ of $R$. prolixus saline (Maddrell et al., 1993). Controls received only saline. Peptides were administered with a $5 \mu \mathrm{l}$ syringe through an incision at the conexive of the first abdominal segment. Due to the incision, and cut wings, the pressure of the injection in each treatment displaces a similar volume of haemolymph, which is eliminated, causing that the final volume remains constant throughout the experiment (Sterkel et al., 2010; VillalobosSambucaro et al., 2015). To minimize the effect of the stress caused by handling, previously to the administration of the first treatment (saline injection), insects were rested for $30 \mathrm{~min}$. The contractions of the aorta and peristaltic waves of the anterior midgut were observed through the dorsal cuticle (segments IV and V of the abdomen) under a dissection microscope. The total number of contractions in a 3-min period was recorded at 5, 15 and 30 min after each dose was applied (Santini and Ronderos, 2007; Sterkel et al., 2010; Villalobos-Sambucaro et al., 2015). All data were collected by the same operator. As in previous studies, forty minutes after the treatments, the frequency of contractions observed resembled the frequency of the control, showing that the insects tend to return to basal conditions (Sterkel et al., 2010). The same individual was used to assay different doses. Results are expressed as number of contractions or peristaltic waves per minute (frequency of contractions).

To further understand the mechanism of action of AST-C, we decided to re-assay the effect of the peptide on recently fed insects in the presence of the inhibitor of the endoplasmic reticulum Calcium-ATPase Thapsigargin (TG) (Treiman et al., 1998). A new group of recently fed insects, previously stabilized with saline, was treated with AST-C $\left(10^{-6} \mathrm{M}\right)$ plus TG $\left(10^{-6} \mathrm{M}\right)$. The number of aorta contractions and peristaltic waves of the crop were recorded at 5, 15 and 30 min after each treatment.

\subsection{Statistical analysis}

Significant differences were evaluated by multifactorial or Repeated Measures Analysis of Variance (ANOVA). Single post hoc comparisons were tested by the LSD test. Each experimental group was constituted by $6-12$ individuals. Only differences equal or less than 0.05 were considered significant. Data are expressed as Means \pm Standard error.

\subsection{Identification of the RpAST-Cr gene}

Based on the sequences of the Tribolium castaneum AST-C receptor (XP_971178.2), the sequence of the corresponding ortholog gene was searched in the $R$. prolixus genome (http://vectorbase. org) using the TBLASTN algorithm and the BLOSUM62 matrix. The structure of the genes (ORF, introns and exons) were predicted using the software Augustus (http://augustus.gobics.de/).

\subsection{Analysis of the sequences}

Sequences analyses were performed using holometabolous and hemimetabolous sequences available in GeneBank. The accession numbers of the AST-C receptor sequences are: XP_003486456.1 (Bombus impatiens), XP_003394391.1 (Bombus terrestris), XP_003698610.1 (Apis florea), XP_006616354.1 (Apis dorsata), XP_006560939.1 (Apis mellifera), XP_003706519.1 (Megachile rotundata), EFN69671.1 (Camponotus floridanus), XP_001600654.2 (Nasonia vitripennis), XP_008548803.1 (Microplitis demolitor), EFN80627.1 (Harpegnathos saltator), EFZ10721.1 (Solenopsis invicta), D6X173_TRICA (Tribolium castaneum), EHJ63490.1 (Danaus plexippus), ADX66345.1 (Manduca sexta), NP_001127736.1 (Bom- 
byx mori), XP_001662510.1 (A. aegypti), XP_001854846.1 (Culex quinquefasciatus), XP_005179255.1 (Musca domestica), M9PFL7_DROME (D. melanogaster), XP_002008619.1 (Drosophila mojavensis), XP_002042582.1 (Drosophila sechellia), XP_001353984.2 (Drosophila pseudoobscura pseudoobscura), XP_002094901.1 (Drosophila yakuba), XP_001972840.1 (Drosophila erecta), AHE41430.1 (R. prolixus), XP_001950448.2 (Acyrthosiphon pisum), BA001050.1 (Nilaparvata lugens), XP_008479092.1 (Diaphorina citri).

The sequences were aligned using the Clustal Wallis algorithm (http://www.ebi.ac.uk/Tools/msa/clustalw2/) and further analyzed by the JalView 2.7 (Waterhouse et al., 2009). Transmembrane domains of the receptors were determined using the online software Interproscan (Jones et al., 2014) and using the TMHMM Server v. 2.0 (http://www.cbs.dtu.dk/services/TMHMM/). Finally, the analysis of evolutionary relationships between sequences was performed using the maximum likelihood method based on the Poisson correction model, including a 1000 replicates bootstrap analysis, by the use of Mega 6.06 software (Tamura et al., 2013).

\subsection{MRNA expression}

To amplify fragments of the $R$. prolixus AST-C receptor (RpAST$C r$ ) transcript, the following primers were designed: Primer Forward 5'-AATCTAAGCGGCCAGACAGCG-3'; Primer Reverse 5'TAGATGTGAGCGCCGTTGTGG-3', corresponding to a 577 bp fragment of RpAST-Cr that was sequenced and annotated; and Primer Forward 5'-AAGCGTGCACTTGTGCTGCTGG-3'; Primer Reverse 5'-A TGTGAGCGCCGTTGTGGAATG-3'.

RNA was isolated from different organs of pooled adults $R$. prolixus collected at different times before and after feeding using the RNeasy kit according to the manufacture's specifications (Qiagen). RNA was treated with RNAse-free DNAse (Qiagen), cDNA was synthesized using Revert Aid First Strand cDNA Synthesis Kit (Fermentas, USA) and used as template in a PCR reaction with the primers indicated above. PCR products were sequenced at the Unidad de Genómica - Instituto de Biotecnología - CICVyA - CNIA - INTA (Argentina).

\section{Results}

3.1. Antagonistic effect of AST-C on the cardio acceleratory activity of AT

AST-C $\left(10^{-6} \mathrm{M}\right)$ was applied to insects after they have reached the maximum increase of dorsal vessel frequency obtained in our experimental system due to consecutive treatments with serotonin $\left(10^{-9} \mathrm{M}\right)$ and AT $\left(10^{-9} \mathrm{M}\right)$. The frequency of contractions of the aorta decreased significantly after treatment with AST-C (Fig. 1A, supplementary movie 1 ). In some individuals, the occurrence of a number of antero-posterior like waves was observed (supplementary movie 1). The frequency of the peristaltic waves of the crop under the same treatments and during the same period did not show statistically significant differences (Fig. 1B). Notably, after treatment with AST-C, the frequency of the contractions of the aorta decreased to a frequency similar to that previously reached by the serotonin treatment, suggesting that AST-C is antagonizing the synergistic effect of AT on tissues previously exposed to serotonin (Fig. 1A). The analysis of the data by Repeated Measures ANOVA showed that the inhibitory effect of AST-C occurred mainly during the first 15 min after injection (Fig. 2A). When AST-C was applied just after the serotonin treatment (i.e. without AT stimulation), the frequency of contractions of the aorta was not modified (Fig. 2B).

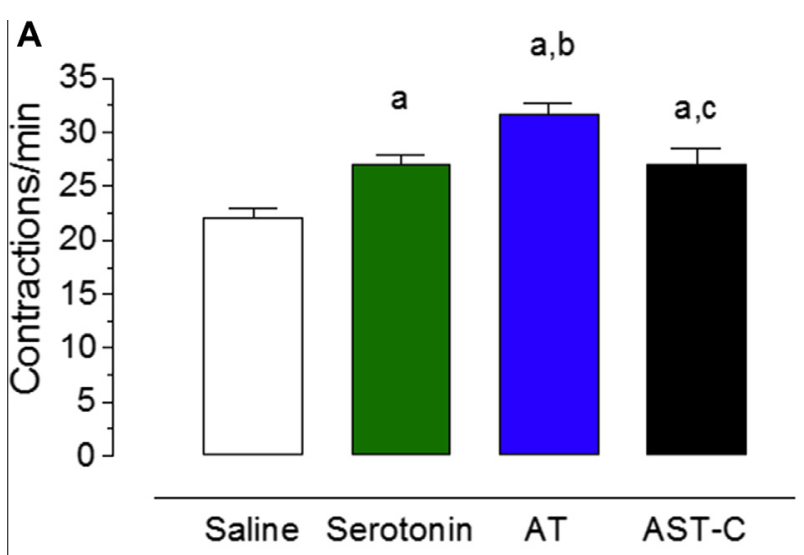

B

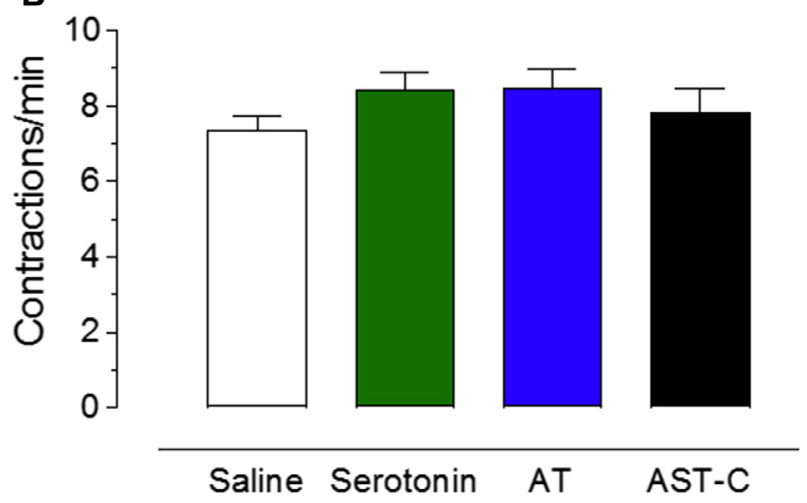

Fig. 1. Antagonistic effect of AST-C on the cardio acceleratory activity of AT. (A) Addition of AST-C $\left(10^{-6} \mathrm{M}\right)$ decreased the frequency of contractions of the aorta after stimulation with serotonin and AT. (B) The number of peristaltic waves of the anterior midgut is not modified. Data analyzed by multifactorial ANOVA. Each bar represents Mean \pm Standard error.

\subsection{Activity of AST-C after blood ingestion}

To further analyze the effect of AST-C we assayed the activity of the peptide during the physiologically critical period of postprandial diuresis. When recently fed insects (i.e. $45 \mathrm{~min}$ after blood ingestion) were treated with different doses of AST-C $\left(10^{-14} \mathrm{M}\right.$, $\left.10^{-12} \mathrm{M}, 10^{-10} \mathrm{M}, 10^{-8} \mathrm{M}, 10^{-6} \mathrm{M}\right)$ the number of contractions of the aorta, as well as of the rate of the peristaltic waves of the crop, decreased in a dose-response manner (Fig. 3A and B).

When recently fed insects were treated with AST-C $\left(10^{-6} \mathrm{M}\right)$ in the presence of TG $\left(10^{-6} \mathrm{M}\right)$, there were not statistically significant changes in the frequency of contractions of the aorta or the peristaltic waves of the crop (Fig. 3C and D).

\subsection{Genomic characterization and expression of the putative AST-C receptor in $R$. prolixus}

We identified and cloned the putative $R$. prolixus AST-C receptor (Rp-AST-Cr) (Fig. 4A). The intronless ORF has $1260 \mathrm{bp}$ and encodes a 419 AA protein (Fig. 4A; supplementary Figs. 1 and 2). The predicted protein includes the seven transmembrane domain characteristics of the receptor family (Fig. 4A and supplementary Fig. 2). A detailed analysis of the sequence shows that Rp-AST-Cr presents the amino acid sequence DRY at the cytoplasmic face of the TM3 that is characteristic in most of GPCRs, including somatostatinlike receptors (Fig. 4B and supplementary Fig. 2). Furthermore, all the conserved features of a somatostatin-like receptor were present, including several $\mathrm{N}$-linked glycosylation sites in the $\mathrm{N}$ terminal domain and several probable palmitoylation sites 
A

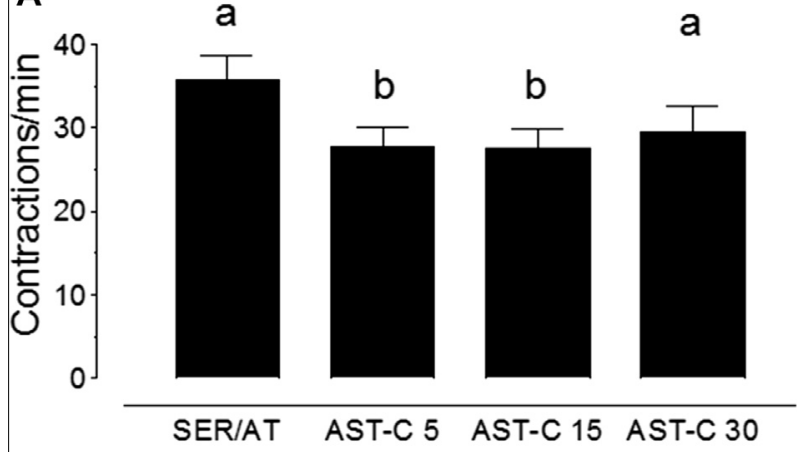

B

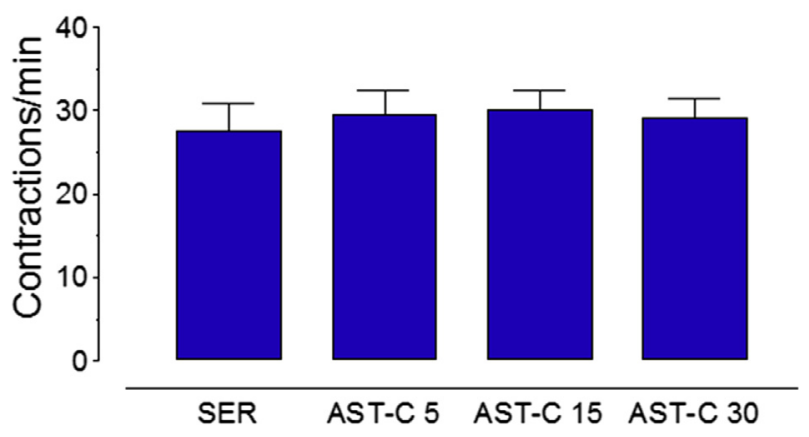

Fig. 2. Time-dependent effect of AST-C on the frequency of contractions of the aorta. (A) The inhibitory effect of AST-C on the frequency of contractions of the aorta applied $30 \mathrm{~min}$ after being stimulated with serotonin/AT was significant during the first $15 \mathrm{~min}$ of the treatment. (B) AST-C had no effect when applied after serotonin treatment. Data analyzed by Repeated Measure ANOVA. Each bar represents Mean \pm Standard error.

(Fig. 4B). In addition, the highly conserved sequence YSNSAMNPILYA is also present (Fig. 4B and supplementary Fig. 2). The alignment of Rp-AST-Cr indicated a high degree of homology with AST-C receptors from other insect species (supplementary Fig. 2).
Transcripts for AST-C were present in all the organs analyzed, including those two relevant for these studies, namely the MG and dorsal vessel (Fig. 4C).

Regarding the phylogenetic relationship with the other sequences of insects species included in the analysis, the sequence corresponding to the putative AST-C receptor of $R$. prolixus resulted well grouped with the other hemimetabola species (i.e. species pertaining to the order Hemiptera). Notably, the species corresponding to the order Hymenoptera (holometabolous) appeared grouped with Hemiptera (hemimetabolous), but not with the rest of holometabolous species (i.e. Diptera, Lepidoptera, Coleoptera) that were clustered in a different branch (Fig. 5).

\section{Discussion}

Previous studies described cardio acceleratory and myostimulatory activities of AT on the crop and HG in R. prolixus (VillalobosSambucaro et al., 2015) and T. infestans (Santini and Ronderos, 2007; Sterkel et al., 2010). The presence of allatotropic nerves innervating aorta, crop and HG in $R$. prolixus and $T$. infestans were also described (Masood and Orchard, 2014; Riccillo and Ronderos, 2010; Sterkel et al., 2010). In T. infestans, AT increased the contractions of the digestive tract (midgut and HG) and dorsal vessel (Santini and Ronderos, 2007; Sterkel et al., 2010). AT regulatory activity on the peristaltic waves of the HG was also confirmed by injecting juvenile individuals with anti-AT antiserum (Santini and Ronderos, 2007). In addition, feeding juvenile and adults individuals of $R$. prolixus with anti-AT antiserum resulted in a decrease in the frequency of contractions of the DV, the peristaltic activity of the crop and the total quantity of urine eliminated by larvae (Villalobos-Sambucaro et al., 2015). AST-C also inhibits foregut contractions in the Lepidoptera Lacanobia oleracea (Duve et al., 2000; Matthews et al., 2007) and heart contractions in D. melanogaster (Price et al., 2002).

Genes encoding AST-C related peptides have been found in several insect groups including hemimetabola such as Orthoptera and Hemiptera, as well as in mites and crustacean species (Veenstra, 2009). Surprisingly, only the sequence defined as its paralogue
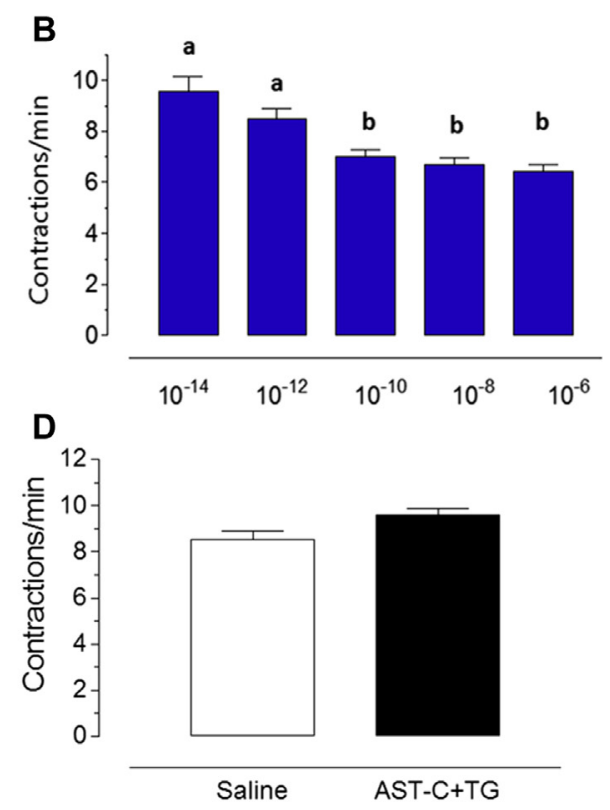

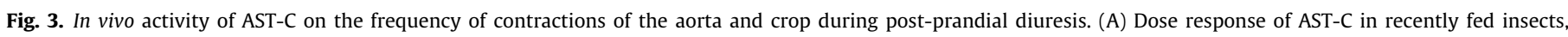

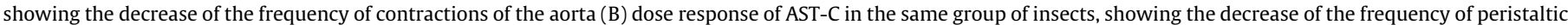

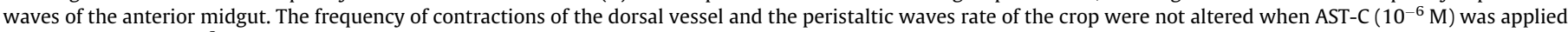
together with TG $\left(10^{-6} \mathrm{M}\right)(\mathrm{C}$ and $\mathrm{D})$. Data analyzed by multifactorial ANOVA. Each bar represents Mean \pm Standard error. 
A

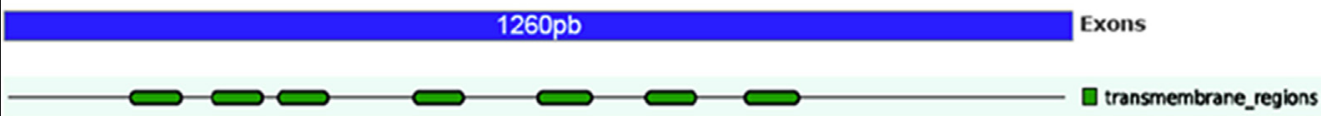

B

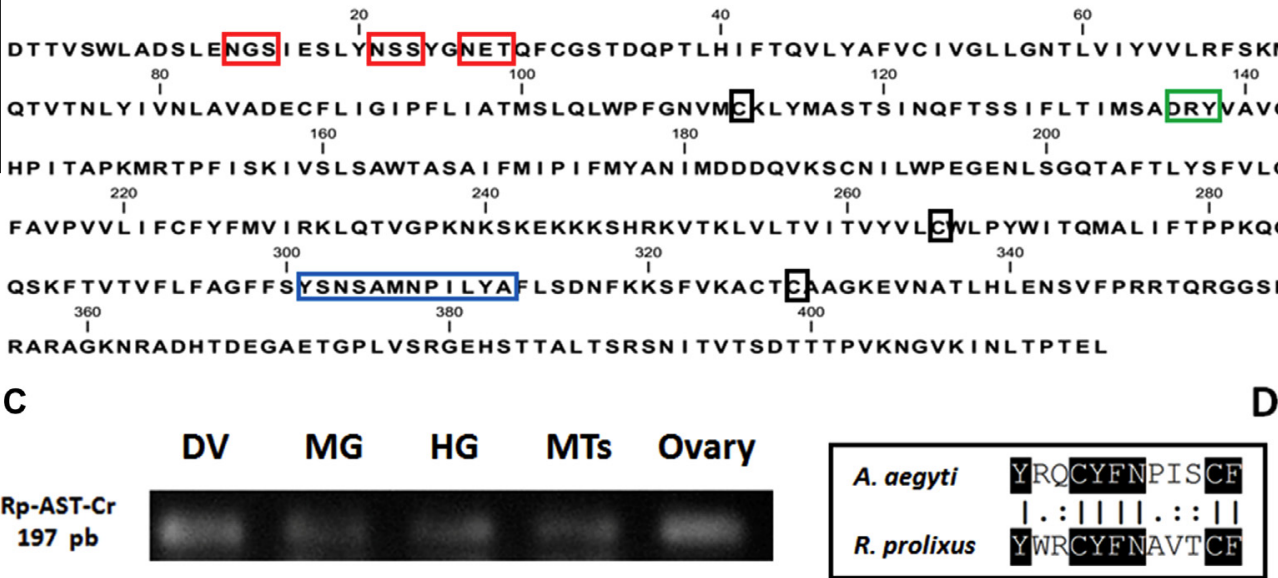

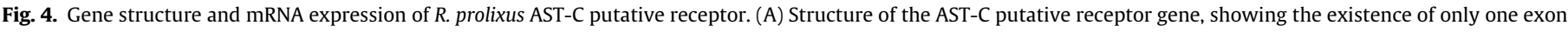

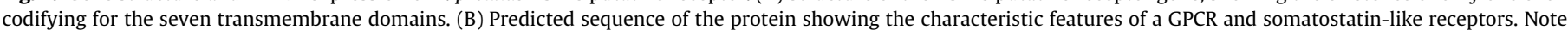

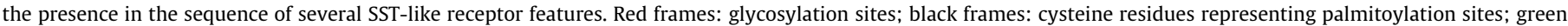

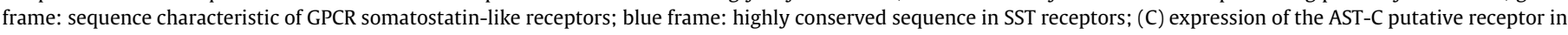

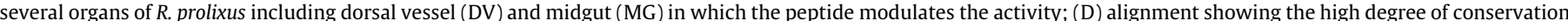

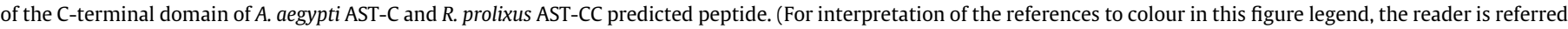
to the web version of this article.)
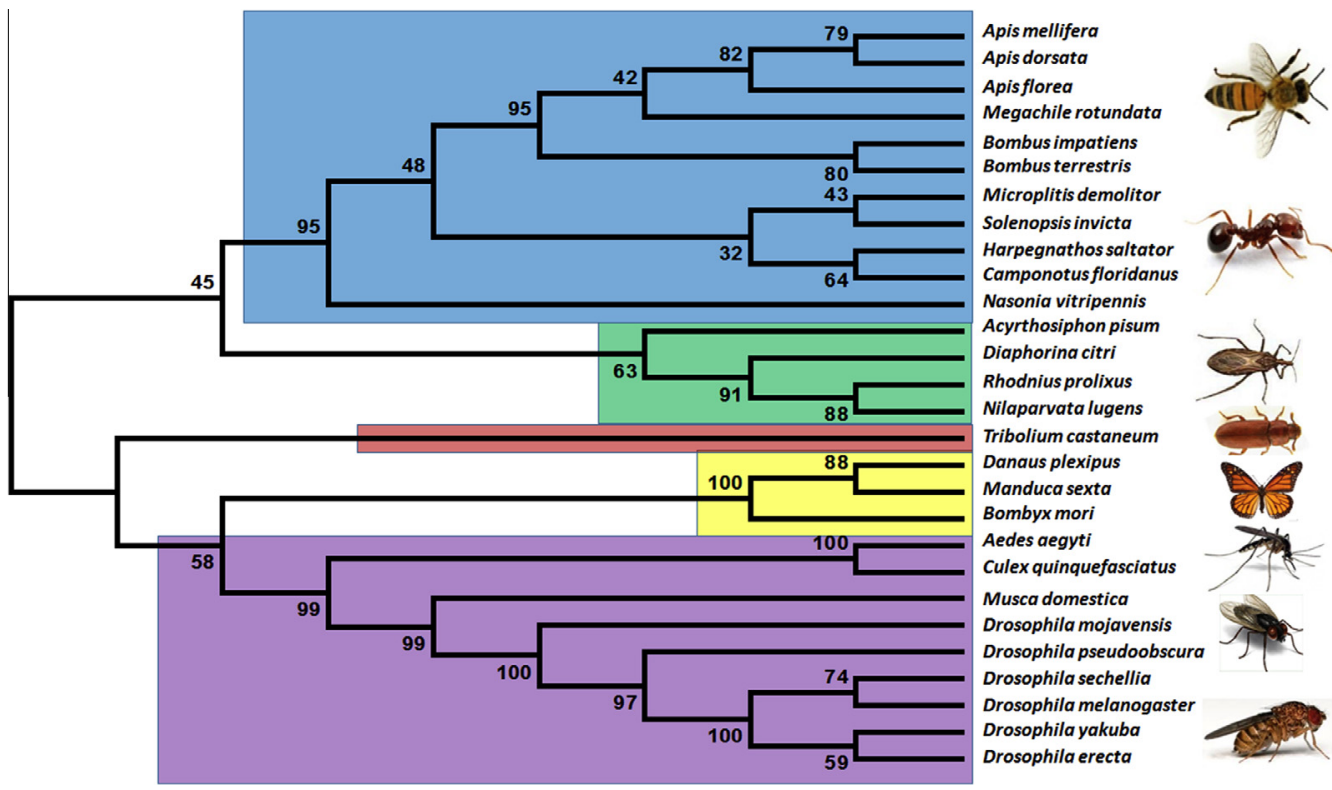

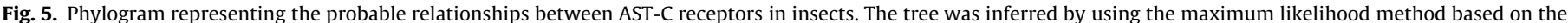

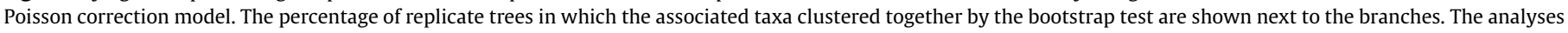
were performed with MEGA 6.06.

(AST-CC) has been annotated in the $R$. prolixus genome (Veenstra, 2009). Comparison of the A. aegypti AST-C used in this study with the predicted sequence of $R$. prolixus AST-CC showed a $58.3 \%$ of identity and $83.3 \%$ of similarity for 12 out of 16 amino acids at the C-terminal of the active peptide (Fig. 4D), suggesting that $A$. aegypti AST-C peptide could effectively bind to the AST-C receptor in $R$. prolixus tissues.
The results presented in this study shows that AST-C decreased contraction frequencies in target tissues to values similar to those observed before the addition of AT (i.e. the frequency after treatment with serotonin). Furthermore, AST-C had no effect when applied just after serotonin treatment, suggesting that this peptide is acting specifically on the synergistic increment caused by AT. 
AST-C had no effect on the peristaltic wave frequencies of the crop under basal conditions, as well as on the crop treated with serotonin and AT in non-fed adult. On the contrary, during postprandial diuresis, AST-C showed a dose-response reduction of aorta beat frequency, as well as peristaltic waves of the crop. These results suggest that AST-C is involved in the regulation of the haemolymph recirculation during post-prandial diuresis, when serotonin and AT are physiologically active (Maddrell et al., 1991; Santini and Ronderos, 2007; Sterkel et al., 2010), acting on the postero-anterior (DV) and antero-posterior direction of the haemolymph flow. The lack of response of the crop in unfed insects suggests that besides serotonin, additional factor/s might be implicated in crop muscle activity regulation.

The existence of a somatostatin-like receptor for AST-C in insects raises the possibility that this peptide shares an evolutionary relationship with vertebrate somatostatin (SST), a neuropeptide originally isolated from the hypothalamus based on its ability to inhibit growth hormone secretion. SST has also pleiotropic functions and inhibits the secretion of several hormones, acting through the activation of five different G-protein-coupled receptors (Patel, 1999), and inducing a hyperpolarization of cell membranes that diminishes the availability of cytosolic $\mathrm{Ca}^{2+}$ (Barbieri et al., 2013; Patel, 1999). It has been proposed that AT acts by inducing an increment in the availability of cytosolic $\mathrm{Ca}^{2+}$ (Lismont et al., 2015; Nouzova et al., 2012; Rachinsky et al., 2003; Verlinden et al., 2013). Interestingly, the use of TG, an inhibitor of the endoplasmic reticulum Calcium-ATPase, counteracted the effect of AST-C, suggesting that the antagonistic effect of this peptide could be due to a depletion of the cytosolic $\mathrm{Ca}^{2+}$. Indeed, AST-C receptors in insects might antagonize AT activity by inducing a membrane hyperpolarization and a decrease of intracellular $\mathrm{Ca}^{2+}$, necessary for muscle contraction.

Several studies had stated that in a number of holometabolous and hemimetabolous insect species, the heart beat alternates between anterograde and retrograde directions (Gerould, 1933; Glenn et al., 2010; League et al., 2015). This alternation was shown to be restricted only to the adult stage in the mosquito A. gambiae (League et al., 2015), but not in triatominae insects (VillalobosSambucaro et al., 2015). In the present study it was found that some individuals undergoing AST-C treatment occasionally evidenced antero-posterior-like contractions of the DV. This behavior seems like an artefactual response that might be induced by changes in cytosolic $\mathrm{Ca}^{2+}$ availability, causing alterations in the normal coordination of the contractions.

The expression of the putative RpAST-Cr in organs involved in haemolymph recirculation during post-prandial diuresis (midgut and DV) is an additional evidence for the regulatory role of the peptide. Furthermore, the expression of the putative receptor in the female reproductive system, which in fact presents a welldeveloped musculature (Sedra and Lange, 2014), suggests that this peptide could be acting as a myoregulator in other organs.

The sequence of the RPAST-Cr displays all the characteristic features of the somatostatin/AST-C family of receptors, including the well conserved YSNSAMNPILYA sequence in the TM7, which is considered a signature of this family (Mayoral et al., 2010; Olias et al., 2004). Maximum likelihood phylogenetic analysis showed that RpAST-Cr clusters together with the sequences corresponding to other species of the order Hemiptera, and notably to Hymenoptera. It has been proposed that while holometabolous insect orders conform a monophyletic group, hemimetabolous are polyphyletic (Belles, 2011; Truman and Riddiford, 1999). Our analysis does not represent a conclusive phylogenetic relationship between Hemiptera and Hymenoptera, and in fact it might be influenced by the limited number of hemimetabolous sequences available.

In summary, our results suggest that the process of postprandial diuresis is facilitated by synergistic and antagonistic actions of serotonin, AT and AST-C, which might play an important role by regulating haemolymph circulation as a result of modulation of aorta contractions and anterior midgut peristaltic waves during this critical physiological process.

\section{Acknowledgments}

This study was supported by a Universidad Nacional de La Plata N673 award to JRR, and an NIH 2R01AI045545-15 to FGN. MJVS is a fellow of UNLP. LD is member of CONICET.

\section{Appendix A. Supplementary data}

Supplementary data associated with this article can be found, in the online version, at http://dx.doi.org/10.1016/j.ygcen.2016.05. 009.

\section{References}

Barbieri, F., Bajetto, A., Pattarozzi, A., Gatti, M., Wurth, R., Thellung, S., Corsaro, A. Villa, V., Nizzari, M., Florio, T., 2013. Peptide receptor targeting in cancer: the somatostatin paradigm. Int. J. Pept. http://dx.doi.org/10.1155/2013/926295.

Belles, X., 2011. Origin and Evolution of Insect Metamorphosis. John Wiley \& Sons, Ltd., Chichester, 10.1002/9780470015902.a0022854.

Bendena, W.G., Tobe, S.S., 2012. Families of allatoregulatory sequences: a 2011 perspective. Can. J. Zool. 90, 521-544.

Buxton, P.A., 1930. The biology of a blood-sucking bug, Rhodnius prolixus. Trans. R. Entomol. Soc. Lond. 78, 227-236.

Chiang, R.G., Chiang, J.A., Davey, K.G., 1990. Morphology of the dorsal vessel in the abdomen of the blood-feeding insect Rhodnius prolixus. J. Morphol. 204, 9-23.

Dasari, S., Cooper, R.L., 2006. Direct influence of serotonin on 1 the larval heart of Drosophila melanogaster. J. Comp. Physiol. B 176, 349-357.

Duve, H., East, P.D., Thorpe, A., 1999. Regulation of lepidopteran foregut movement by allatostatins and allatotropin from the frontal ganglion. J. Comp. Neurol. 413, 405-416.

Duve, H., Audsley, N., Weaver, R.J., Thorpe, A., 2000. Triple co-localization of two types of allatostatin and an allatotropin in the frontal ganglion of the lepidopteran Lacanobia oleracea (Noctuidae): innervation and action on the foregut. Cell Tissue Res. 300, 153-163.

Gerould, J.H., 1933. Orders of insects with heart-beat reversal. Biol. Bull. 64, 424431.

Glenn, J.D., King, J.G., Hillyer, J.F., 2010. Structural mechanics of the mosquito heart and its function in bidirectional hemolymph transport. J. Exp. Biol. 213, 541550 .

Hernández-Martínez, S., Li, Y., Lanz-Mendoza, H., Rodriguez, M.H., Noriega, F.G. 2005. Immunostaining for allatotropin and allatostatin- $A$ and $-C$ in the mosquitoes Aedes aegyti and Anopheles albimanus. Cell Tissue Res. 321, 105-113.

Hillyer, J.F., Estévez-Lao, T.Y., Mirzai, H.E., 2015. The neurotransmitters serotonin and glutamate accelerate the heart rate of the mosquito Anopheles gambiae. Comp. Biochem. Physiol. A 188, 49-57.

Jones, P., Binns, D., Chang, H.Y., Fraser, M., Li, W., McAnulla, C., McWilliam, H., et al., 2014. InterProScan 5: genome-scale protein function classification. Bioinformatics 30, 1236-1240.

Kataoka, H., Toschi, A., Li, J.P., Carney, R.L., Schooley, D.A., Kramer, S.J., 1989. Identification of an allatotropin from adult Manduca sexta. Science 243, 14811483.

Koladich, P.M., Cusson, M., Bendena, W.G., Tobe, S.S., McNeil, J.N., 2002 Cardioacceleratory effects of Manduca sexta allatotropin in the true armyworm moth Pseudaletia unipuncta. Peptides 23, 645-651.

League, G.P., Onuh, O.C., Hillyer, J.F., 2015. Comparative structural and functional analysis of the larval and adult dorsal vessel and its role in hemolymph circulation in the mosquito Anopheles gambiae. I. Exp. Biol. 218, 370-380.

Lismont, E., Vleugels, R., Marchal, E., Badisco, L., Van Wielendaele, P., Lenaerts, C., Zels, S., Tobe, S.S., Vanden Broeck, J., Verlinden, H., 2015. Molecular cloning and characterization of the Allatotropin precursor and receptor in the desert locust, Schistocerca gregaria. Front. Neurosci. 9, 84. http://dx.doi.org/10.3389/ fnins.2015.00084.

Maddrell, S.H.P., 1964. Excretion in the blood-sucking bug Rhodnius prolixus Stal. II The normal course of diuresis and the effect of temperature. J. Exp. Biol. 41 163-176.

Maddrell, S.H.P., 1978. Physiological discontinuity in an epithelium with an apparently uniform structure. J. Exp. Biol. 75, 133-145.

Maddrell, S.H.P., Phillips, J.E., 1975. Secretion of hypo-osmotic fluid by the lower Malpighian tubules of Rhodnius prolixus. J. Exp. Biol. 62, 671-683.

Maddrell, S.H.P., Herman, W.S., Mooney, R.L., Overton, J.A., 1991. 5hydroxytryptamine: a second diuretic hormone in Rhodnius prolixus. J. Exp. Biol. 156, 557-566.

Maddrell, S.H.P., O’Donnell, M.J., Caffrey, R., 1993. The regulation of haemolymph potassium activity during initiation and maintenance of diuresis in fed Rhodnius prolixus. J. Exp. Biol. 177, 273-285. 
Masood, M., Orchard, I., 2014. Molecular characterization and possible biologica roles of allatotropin in Rhodnius prolixus. Peptides 53, 159-171.

Matthews, H.J., Audsley, N., Weaver, R.J., 2007. Interactions between allatostatins and allatotropin on spontaneous contractions of the foregut of larval Lacanobia oleracea. J. Insect Physiol. 53, 75-83.

Mayoral, J.G., Nouzova, M., Brockhoff, A., Goodwin, M., Hernández-Martínez, S., Richter, D., Meyerhof, W., Noriega, F.G., 2010. Allatostatin-C receptors in mosquitoes. Peptides 31, 442-450.

Nouzova, M., Brockhoff, A., Mayoral, J.G., Goodwin, M., Meyerhof, W., Noriega, F.G. 2012. Functional characterization of an allatotropin receptor expressed in the corpora allata of mosquitoes. Peptides 34, 201-208.

O’Donnell, M.J., Ianowski, J.P., Linton, S.M., Rheault, M.R., 2003. Inorganic and organic anion transport by insect renal epithelia. Biochim. Biophys. Acta 1618 194-206.

Olias, G., Viollet, C., Kusserow, H., Epelbaum, J., Meyerhof, W., 2004. Regulation an functions of somatostatin receptors. J. Neurochem. 89, 1057-1091.

Orchard, I., 2006. Serotonin: a coordinator of feeding-related physiological events in the blood-gorging bug, Rhodnius prolixus. Comp. Biochem. Physiol. A 144, $316-$ 324.

Paluzzi, J.-P.V., Bhatt, G., Wang, C.-H.J., Zandawala, M., Lange, A.B., Orchard, I., 2015 Identification, functional characterization, and pharmacological profile of a serotonin type-2b receptor in the medically important insect, Rhodnius prolixus. Front. Neurosci. 9, 175.

Patel, Y.C., 1999. Somatostatin and its receptor family. Front. Neuroendocrinol. 20, 157-198.

Price, M.D. Merte, I. Nichols, R., Koladich, P.M. Tobe, S.S., Bendena, W.G., 2002 Drosophila melanogaster flatline encodes a myotropin orthologue to Manduca sexta allatostatin. Peptides 23, 787-794.

Rachinsky, A., Srinivasan, A., Ramaswamy, S.B., 2003. Regulation of juvenile hormone biosynthesis in Heliothis virescens by Manduca sexta Allatotropin. Arch. Insect Biochem. Physiol. 54, 121-133.

Ramsay, J.A., 1952. The excretion of sodium and potassium by the Malpighian tubules of Rhodnius. J. Exp. Biol. 29, 110-126.

Riccillo, F.L., Ronderos, J.R., 2010. Allatotropin expression during the development of the fourth instar larvae of the kissing-bug Triatoma infestans (Klüg). Tissue Cell 42, 355-359.
Robertson, L., Rodriguez, E.P., Lange, A.B., 2012. The neural and peptidergic control of gut contraction in Locusta migratoria: the effect of an FGLa/AST. J. Exp. Biol. 215, 3394-3402.

Rudwall, A.J., Sliwowska, J., Nässel, D.R., 2000. Allatotropin-like neuropeptide in the cockroach abdominal nervous system: myotropic actions, sexually dimorphic distribution and colocalization with serotonin. J. Comp. Neurol. 428, 159-173.

Santini, M.S., Ronderos, J.R., 2007. Allatotropin-like peptide released by Malpighian tubules induces hindgut activity associated to diuresis in the Chagas disease vector Triatoma infestans (Klüg). J. Exp. Biol. 210, 1986-1991.

Sedra, L., Lange, A.B., 2014. The female reproductive system of the kissing bug, Rhodnius prolixus: arrangements of muscles, distribution and myoactivity of two endogenous FMRFamide-like peptides. Peptides 53, 140-147.

Sterkel, M., Riccillo, F.L., Ronderos, J.R., 2010. Cardioacceleratory and myostimulatory activity of allatotropin in Triatoma infestans (Klüg). Comp. Biochem. Physiol. A 155, 371-377.

Tamura, K., Stecher, G., Peterson, D., Filipski, A., Kumar, S., 2013. MEGA6: molecular evolutionary genetics analysis version 6.0. Mol. Biol. Evol. 30, 2725-2729.

Treiman, M., Caspersen, C., Christensen, S.B., 1998. A tool coming of age: thapsigargin as an inhibitor of sarcoendoplasmic reticulum $\mathrm{Ca}^{2+}$-ATPases. Trends Pharmacol. Sci. 19, 131-135.

Truman, J.W., Riddiford, L., 1999. The origins of insect metamorphosis. Nature 401, 447-452.

Veenstra, J.A., 2009. Allatostatin C and its paralog allatostatin double C: the arthropod somatostatins. Insect Biochem. Mol. Biol. 39, 161-170.

Verlinden, H., Lismont, E., Bil, M., Urlacher, E., Mercer, A., Vanden Broeck, J., Huybrechts, R., 2013. Characterization of a functional allatotropin receptor in the bumblebee, Bombus terrestris (Hymenoptera, Apidae). Gen. Comp. Endocrinol. 193, 193-200.

Villalobos-Sambucaro, M.J., Lorenzo-Figueiras, A.N., Riccillo, F.L., Diambra, L.A. Noriega, F.G., Ronderos, J.R., 2015. Allatotropin modulates myostimulatory and cardioacceleratory activities in Rhodnius prolixus (Stal). PLoS One 10, e0124131.

Waterhouse, A.M., Procter, J.B., Martin, D.M.A., Clamp, M., Barton, G.J., 2009. JalView version 2 - a multiple sequence alignment editor and analysis workbench. Bioinformatics 25, 1189-1191. 